\title{
Relationship between thyroid dysfunction and body weight: a not so evident paradigm
}

This article was published in the following Dove Press journal:

International Journal of General Medicine

\author{
Mónica Ríos-Prego' \\ Luis Anibarro' \\ Paula Sánchez-Sobrino ${ }^{2}$ \\ 'Department of Internal Medicine, \\ Pontevedra University Hospital Complex, \\ Pontevedra, Spain; ${ }^{2}$ Department of \\ Endocrinology and Nutrition, Pontevedra \\ University Hospital Complex, \\ Pontevedra, Spain
}

Purpose: Hypothyroidism has traditionally been associated with obesity, whereas hyperthyroidism has been linked to being underweight. However, very few studies have assessed these associations. The aim of this work is to evaluate the association between thyroid dysfunction and body mass index (BMI) at baseline and after normalization of the hormone levels.

Patients and methods: A retrospective, observational study of a cohort of otherwise healthy patients that were referred for evaluation of thyroid dysfunction to the Endocrine Department of Pontevedra University Complex Hospital, Spain was conducted. We collected data of BMI and thyroid hormone levels before treatment and after normalization of thyroid function within a follow-up period of 12 months.

Results: A total of 330 patients were initially selected for the study. In order to exclude variables that for any reason could influence on BMI, 235 were excluded for further studies. Another 61 patients were also excluded because incomplete data on their medical records, failure to achieve euthyroidism, or lost to follow-up. Therefore, the eligible final study group consisted of 34 patients (17 with hypothyroidism and 17 with hyperthyroidism). No differences were observed in mean baseline BMI between hypo and hyperthyroid patients $(27.07 \pm 3.22$ vs $26.39 \pm 4.44, p=0.609)$. Overweight or obesity was observed in $76.5 \%$ and $58.8 \%$ of hypothyroid and hyperthyroid patients, respectively ( $p=0.23$ ). After normalization of thyroid function, the weight of hypothyroid patients decreased from $70.93 \pm 10.06 \mathrm{~kg}$ to $68.68 \pm 10.14(p=0.000)$, while the weight of hyperthyroid patients increased from $65.45 \pm 11.64 \mathrm{~kg}$ to $68.37 \pm 12.80(p=0.000)$. Their mean BMI was 26.22 \pm 3.36 and $27.57 \pm 4.98$ ( $p=0.361$ ) for hypo- and hyperthyroid patients, respectively. $58.8 \%$ and $64.7 \%$ patients remained in the overweight/obesity range in each group $(p=0.72)$.

Conclusion: Untreated thyroid dysfunction is not associated with BMI. Normalization of thyroid levels significantly changed the weight of patients, but remaining most patients within overweight ranges.

Keywords: hypothyroidism, hyperthyroidism, body mass index, obesity

\section{Introduction}

Thyroid disorders are highly prevalent diseases affecting nearly $10 \%$ of the population in Spain. ${ }^{1}$ In a recent study in adults, the prevalence of hypothyroidism was $9.1 \%$ and $0.8 \%$ for hyperthyroidism. ${ }^{1}$ Thyroid disorders are the major cause of referral to the Endocrinology Department in our Hospital, reaching $47.6 \%$ of all first visits (unpublished data).

Thyroid hormones are involved in multiple physiological processes and regulating basal metabolic rate, promote the adrenergic nervous system to generate heat in response to cold exposure, stimulate gluconeogenesis and both lipolysis and lipogenesis. ${ }^{2}$ Patients with thyroid dysfunction may experience changes in body weight and body composition. ${ }^{3}$
Correspondence: Paula Sánchez-Sobrino Department of Endocrinology and Nutrition, Pontevedra University Hospital Complex, Hospital Montecelo, Mourente sn, Pontevedra 3607I, Spain

Tel +34986800132

Email paula_ss_82@hotmail.com 
Hypothyroidism induces a decreased basal metabolism and thermogenesis, an accumulation of hyaluronic acid and a decreased renal flow, all factors leading to water retention. Severe hypothyroidism states lead to a clinical picture known as myxoedema in which hyperkeratosis of the skin and facial edema could give the patient a false appearance of overweight. ${ }^{4,5}$ Patients with hypothyroidism have also slow peristalsis causing chronic constipation that may result in weight gain. This weight gain is mainly due to water retention and is not related to an increase in fat mass. ${ }^{3}$ In addition, some studies have found a higher prevalence of subclinical hypothyroidism among obese patients. ${ }^{6}$ Altogether, these factors have probably contributed to a general belief of a direct association of hypothyroidism with obesity. The American Thyroid Association in the 2012 guidelines on the management of hypothyroidism makes reference to the absence of evidence in this field. These guidelines mention that hypothyroidism and obesity are often linked at least in the consciousness of the lay public, and that although some observational studies correlate thyrotropin (TSH) levels with body mass index (BMI) others do not. ${ }^{7}$ To our knowledge, very few studies have directly assessed this topic and no clear association has been established between hypothyroidism and obesity. ${ }^{8}$

Conversely, hyperthyroidism has traditionally been associated with weight loss and underweight. ${ }^{8}$ Subjects with hyperthyroidism have an adrenergic hyperstimulation with increased basal metabolism and thermogenesis, and a greater overall energy expenditure resulting in a tendency toward weight loss. Hyperthyroidism can also induce an increased gastrointestinal transit and occasionally anorexia due to the anorexigenic effect of triiodothyronine. ${ }^{4}$ All these factors may have lead to the belief of a direct association of hyperthyroid states with low weight. ${ }^{9}$ But, as it happens with the case of hypothyroidism, this relationship has not been clearly established.

Based on these alterations, and from a theoretical point of view, patients with thyroid dysfunction would be expected to return to their body weight prior to the onset of the dysfunction once achieving normal hormone levels with specific therapy. However, several studies have shown that this is not always true. ${ }^{10-14}$

The aim of our study was to investigate the association between thyroid dysfunction and BMI at baseline and after normalization of the hormone levels.

\section{Materials and methods}

We carried out a retrospective, observational, descriptive study on a cohort of patients with thyroid dysfunction that were referred to the Department of Endocrinology at the Pontevedra University Hospital Complex in Spain. Patients were followed-up until results of their thyroid function tests were normal within a 12-month period. The medical records of all patients were reviewed.

The variables collected were gender, age, thyroid dysfunction etiology, TSH levels, free levothyroxine (FT4) levels, presence of thyroid autoimmunity (considered as detection of anti-thyroperoxidase antibodies and/or TSH receptor antibodies), BMI and weight at the time of referral to the Department of Endocrinology and after normalization of thyroid hormone levels after treatment.

\section{Inclusion criteria}

Patients over 18 years of age were referred to the Department of Endocrinology for thyroid dysfunction evaluation.

\section{Exclusion criteria}

We excluded for the analysis patients with any variable that for any reason could influence on BMI: Hypocalorific diets, tobacco use, recent quit smoking or recreational drug use, severe psychiatric illness, pregnancy and postpartum stages, diabetes mellitus treated with insulin therapy, sulfonylureas, GLP-1 analogues or SGLT-2 inhibitors, prescription of drugs affecting body weight (corticosteroids, antipsychotics, mirtazapine, anticonvulsants, antineoplastic hormone therapy, fluoxetine or topiramate) and other comorbidities such as advanced congestive heart failure, chronic obstructive pulmonary disease, end-stage renal disease or active neoplasm.

\section{Statistical analysis}

Statistical analysis was performed using the SPSS software version 16 (SPSS Inc., Chicago, Illinois, USA). Categorical variables were expressed in terms of frequency and percentage, and quantitative variables were expressed as mean and standard deviation, or median and rank in cases of a non-normal distribution. The chi-Square or Fisher's test was used to find the association between categorical variables. Normality of quantitative variables was verified using the Kolmogorov-Smirnov test. Quantitative variables were compared using the Student's $t$-test for variables with a normal distribution and the Wilcoxon test for the remaining variables. Differences were considered to be statistically significant when $p<0.05$. 
The study was approved by the Galician Research Ethics Committee (Ref. Code 2015/142). Patients' consent to review their medical records was not required by means of a waiver from the Galician Research Ethics Committee. Due to the retrospective nature of the study and the high number of patients we were prevented from obtaining individual consent. Data confidentiality was preserved and the study is compliant with the Declaration of Helsinki.

\section{Results}

A total of 330 patients were referred to the Department of Endocrinology for thyroid dysfunction evaluation during the study period. Of these, 235 patients were excluded from further analysis for the following reasons: 69 had already initiated treatment for thyroid dysfunction before their first visit to the Department of Endocrinology, 103 were pregnant, 23 were experiencing transient thyroid dysfunction, treatment was not indicated in 18, and 22 were excluded for other reasons. Of the remaining 95 patients, 29 were lost to follow-up, 18 had incomplete medical records, and euthyroidism could not be achieved in the other 14, despite treatment (Figure 1).

Finally, a total of 34 otherwise healthy patients were included for analysis, comprising 6 male $(17.6 \%)$ and 28 female (82.4\%); of these, there were 17 with hyperthyroidism and 17 with hypothyroidism. The mean age was $53.4 \pm 20.4$ years. The demographic, anthropometric, and biochemical characteristics for these patients are shown in Table 1.

The etiology of hypothyroidism per case was 13 patients with primary hypothyroidism (anti-thyroperoxidase antibodies present in eight cases), two with amiodarone-induced hypothyroidism, one with post-surgical hypothyroidism, and one postradioiodine therapy. Seven patients presented subclinical hypothyroidism (TSH $<10 \mu \mathrm{U} / \mathrm{mL}$ ) and two presented severe hypothyroidism (TSH $91.00 \mu \mathrm{U} / \mathrm{mL}$ and $46.50 \mu \mathrm{U} / \mathrm{mL}$, respectively).

The etiology of hyperthyroidism per case was nine patients with Graves-Basedow disease (all with the presence of TSH receptor antibodies), five with Plummer's disease or toxic multinodular goiter, one with primary autoimmune hyperthyroidism, one as a secondary condition to the JodBasedow phenomenon, and one with unknown etiology.

Thyroid autoimmunity was present in 19 subjects, more than $50 \%$ of the sample, according to the two major causes of hypo and hyperthyroidism.

There were no statistically significant baseline differences between the two groups in gender $(p=0.175)$, autoimmunity etiology of the disease $(p=0.300)$, age $(p=0.386)$, or height ( $p=0.086$ ). As expected, significant differences were observed in baseline levels of FT4 and TSH $(p<0.001)$.

When comparing basal mean weight or BMI, no significant differences were found between hypothyroid and hyperthyroid patients: Basal weight $70.93 \pm 10.06 \mathrm{~kg}$ vs

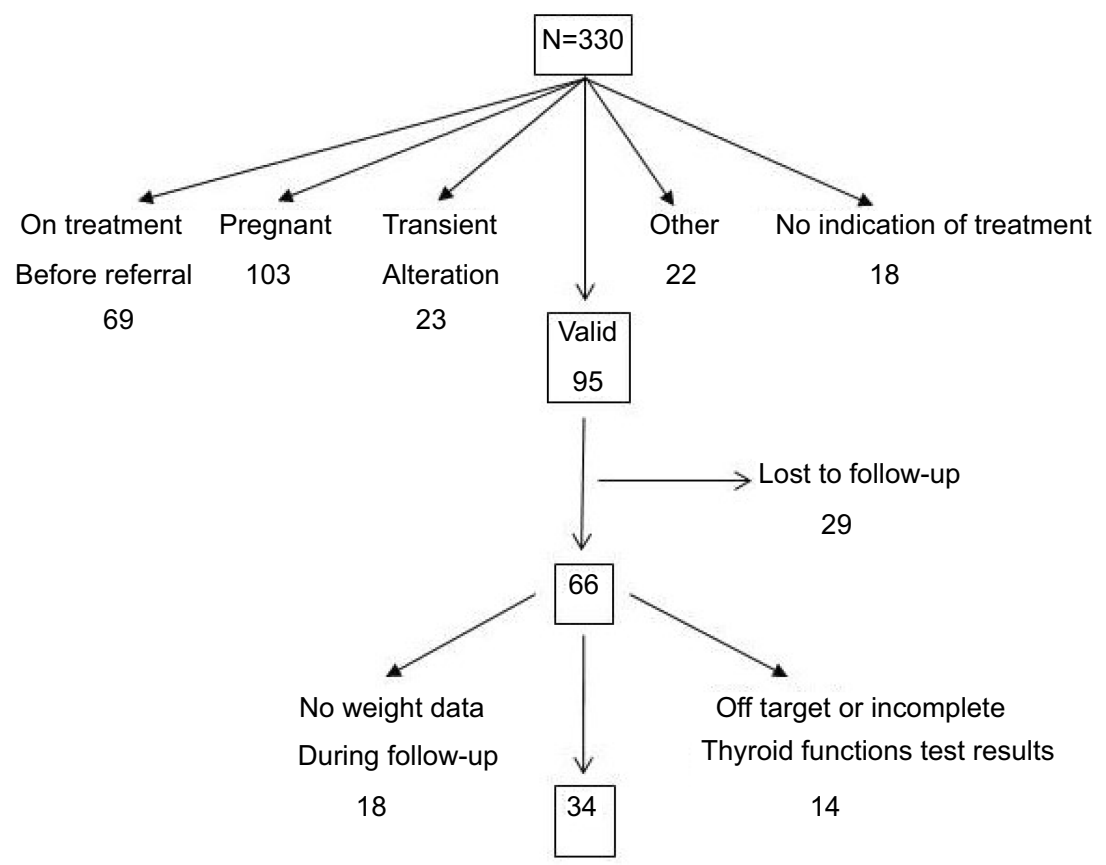

Figure I Patient selection flowchart. 
Table I Baseline and post-treatment demographic, anthropometric, and biochemical characteristics of patients with thyroid dysfunction

\begin{tabular}{|c|c|c|c|c|}
\hline & Total & Hypothyroid patients & Hyperthyroid patients & $p$ \\
\hline N & 34 & 17 & 17 & \\
\hline Gender & 28 female $(82.4 \%)$ & 12 female $(70.6 \%)$ & 16 female $(94.1 \%)$ & 0.175 \\
\hline Age & $53.4 \pm 20.4$ & $55.8 \pm 18.7$ & $50.3 \pm 22.4$ & 0.386 \\
\hline Height $(\mathrm{cm})$ & & $|6| .82 \pm 0.08$ & $157.53 \pm 0.07$ & 0.086 \\
\hline Baseline weight (kg) & & $70.93 \pm 10.06$ & $65.45 \pm 11.64$ & 0.152 \\
\hline Final weight $(\mathrm{kg})$ & & $68.68 \pm 10.14$ & $68.37 \pm 12.80$ & 0.938 \\
\hline Baseline BMI $\left(\mathrm{kg} / \mathrm{m}^{2}\right)$ & & $27.07 \pm 3.22$ & $26.39 \pm 4.44$ & 0.609 \\
\hline Final BMI $\left(\mathrm{kg} / \mathrm{m}^{2}\right)$ & & $26.22 \pm 3.36$ & $27.57 \pm 4.98$ & 0.361 \\
\hline Baseline TSH levels & & $23.99 \pm 26.35$ & $0.03 \pm 0.02$ & 0.000 \\
\hline Final TSH levels & & $3.27 \pm 1.53$ & $2.98 \pm 1.45$ & 0.576 \\
\hline Baseline T4 levels & & $0.67 \pm 0.17$ & $1.58 \pm 0.58$ & 0.000 \\
\hline Final T4 levels & & $0.91 \pm 0.12$ & $0.73 \pm 0.23$ & 0.009 \\
\hline Thyroid autoimmunity & 19 (55.9\%) & $8(47.1 \%)$ & II (64.7\%) & 0.300 \\
\hline ATPO & & $8(47.1 \%)$ & $2(11.7 \%)$ & \\
\hline TSHrAb & & & $9(52.9 \%)$ & \\
\hline
\end{tabular}

Notes: TSH: Thyrotropin. Normal range: $0.34-5.6 \mu \mathrm{U} / \mathrm{mL}$. T4: thyroxine. Normal range: $0.54-1.24 \mu \mathrm{U} / \mathrm{mL}$. ATPO: anti-thyroperoxidase antibodies. TSHr Ab: Anti TSH receptors antibodies.

Abbreviations: BMI, body mass index; FT4, free thyroxine.

$65.45 \pm 11.64 \mathrm{~kg}(p=0.152)$ and BMI $27.07 \pm 3.22 \mathrm{~kg} / \mathrm{m}^{2}$ vs $26.39 \pm 4.44 \mathrm{~kg} / \mathrm{m}^{2}(p=0.609)$ for hypo- and hyperthyroid patients, respectively.

After specific treatment and normalization of thyroid hormone levels, there were still no statistically significant differences in body weight between the two groups $(68.68 \pm 10.14 \mathrm{~kg}$ vs $68.37 \pm 12.80 \mathrm{~kg} ; p=0.938)$ or in BMI $\left(26.22 \pm 3.36 \mathrm{~kg} / \mathrm{m}^{2}\right.$ vs $\left.27.57 \pm 4.98 \mathrm{~kg} / \mathrm{m}^{2} p=0.361\right)$ for hypo- and hyperthyroid patients, respectively. 58.8\% and $64.7 \%$ of the patients remained in the overweight/ obesity range in each group $(p=0.72)$.

After treatment and normalization of thyroid function of the 17 hypothyroid patients, the mean patient weight fell from $70.93 \pm 10.06 \mathrm{~kg}$ to $68.68 \pm 10.14 \mathrm{~kg}$ (mean difference $2.25 \pm 2.01 \mathrm{~kg} ; p<0.001)$. Statistically significant differences were also observed between patients' baseline BMI, $27.07 \pm 3.22 \mathrm{~kg} / \mathrm{m}^{2}$, and final BMI, $26.22 \pm 3.36 \mathrm{~kg} /$ $\mathrm{m}^{2}$ (mean difference $0.86 \pm 0.77 ; p<0.001$ ).

After treatment and normalization of thyroid function of the 17 patients with hyperthyroidism, the mean baseline weight increased from $65.45 \pm 11.64 \mathrm{~kg}$ to $68.37 \pm 12.80 \mathrm{~kg}$, giving a mean difference of $2.92 \pm 3.01 \mathrm{~kg}(p=0.001)$. Statistically significant differences were also observed between the patient's baseline BMI, $26.39 \pm 4.44 \mathrm{~kg} / \mathrm{m}^{2}$, and post-treatment BMI, $27.57 \pm 4.98 \mathrm{~kg} / \mathrm{m}^{2}$ (mean difference $1.18 \pm 1.22 ; p=0.001$ ).

\section{Discussion}

In our study, no statistically significant differences were observed in the weight or the BMI of hypo- and hyperthyroid patients, neither at diagnosis, nor following normalization of hormone levels after treatment. At baseline, both hypo- and hyperthyroid patient groups demonstrated a non-significant weight difference of approximately $6 \mathrm{~kg}$, and a very similar BMI, respectively, with both groups falling into the overweight range. After treatment and normalization of thyroid function, the difference in weight between the two groups was further reduced. The BMI values also remained similar for both groups.

In addition, after analyzing the two different types of thyroid function disorder (hyper- and hypothyroidism) separately, we observed statistically significant differences between baseline and post-treatment body weight and BMI. Hypothyroid patients experienced a mean weight loss of $2.25 \pm 2.01 \mathrm{~kg}$, which is much less than the weight loss described in classic series. ${ }^{6}$ However, we are unaware of the existence of any recent series that had addressed this topic. Our results may have been influenced by the fact that seven of the hypothyroid patients showed a subclinical hormone alteration (TSH $<10 \mu \mathrm{U} / \mathrm{mL}$ ). However, it is noteworthy that patients with a greater degree of hypothyroidism (TSH 91.00 and $46.50 \mu \mathrm{U} / \mathrm{mL}$ ) experienced a weight loss of less than $2 \mathrm{~kg}$ when they reached euthyroid status. 
Some authors suggest that weight gain is greater in post-surgical hypothyroidism than in autoimmune hypothyroidism. ${ }^{12}$ In our study, there was only one case of post-surgical hypothyroidism; this might account for the non-significant weight loss experienced by these patients.

The relationship between hypothyroidism and obesity has historically been based on studies with a baseline population selected from obese patients whose thyroid hormone levels were assessed. In several of these studies, higher incidences of hypothyroidism were found in obese patients than in the general population. ${ }^{16,17,18}$ Based on these findings, some authors have suggested that minor thyroid dysfunction might contribute to significant changes in body weight that could represent a risk factor for overweight and obesity. ${ }^{19}$ However, these studies are inconclusive in establishing a cause-effect relationship, as the demonstration of a higher incidence of hypothyroidism in obese patients does not necessarily mean that the obesity is caused by the disorder. The relationship between hypothyroidism and obesity seems to be weaker than previously thought, even more in subjects with treated hypothyroidism.

In the hyperthyroidism patients group, weight gain was observed on completion of treatment when the results of the thyroid function test returned to normal. This is a common finding in the literature ${ }^{13,15,20,21}$ and there are several theories regarding it. Hyperthyroidism induces an increased basal energy expenditure that leads to weight loss as a result of a decrease in the body's lean and fat mass. When euthyroidism is regained, weight gain occurs at the expense of both compartments. ${ }^{20}$ Another possible explanation for the tendency of hyperthyroid patients to gain weight after achieving euthyroidism could be related to an increase in post-treatment TSH levels over baseline levels. Hence, this would indicate the presence of relative hypothyroidism within the limits of analytic normality. ${ }^{14}$ In our study, we were unable to test this hypothesis because of unavailability of patients' TSH levels prior to the onset of hyperthyroidism.

Our study has a number of limitations that should be taken into account. One of them is that due to the retrospective nature of the study, we could not access information on patients' TSH levels and weight prior to their developing thyroid dysfunction. In the same line, other chemistry data (for example glucose and cholesterol) were not available. Another limitation to our study derives from the small number of patients finally included for analysis. Weight is a variable that can be influenced by multiple factors; due to this, more than $70 \%$ of the patients initially included had to be excluded because of confounding variables that could affect weight, specifically gestation and postpartum states, severe diseases and drugs with proven repercussion on weight. However, this aspect also minimized possible biases. The reason for the high volume of pregnant patients is that the study was conducted before having specific TSH ranges for pregnancy in our population, so we used the ATA ranges. In this context, more than a third of pregnant women were above the upper limit. Thereafter, the upper limit of TSH in pregnancy in our population was calculated resulting in $4 \mu \mathrm{U} / \mathrm{mL}$, with the consequent fewer referrals. Otherwise, patients with stable mild chronic diseases were not excluded (for example, diabetic subjects well controlled with metformin). Another limitation is that composition body data were not recorded, nor by physical measurements like waist perimeter neither impedance techniques.

\section{Conclusion}

In conclusion, hypothyroid and hyperthyroid patients after treatment and normalization of thyroid function have statistically significant changes in BMI, but these do not show great relevance in clinical practice because the BMI remained in the overweight range in both groups.

\section{Disclosure}

The authors report no conflicts of interest in this work.

\section{References}

1. Valdes S, Maldonado-Araque C, Lago-Sampedro A, et al. Populationbased national prevalence of thyroid dysfunction in Spain and associated factors: diabetes study. Thyroid. 2017;27(2):156-166. doi:10.1089/thy.2016.0353

2. Liu YY, Brent GA. Thyroid hormone crosstalk with nuclear receptor signaling in metabolic regulation. Trends Endocrinol Metab. 2010;21 (3):166-173. doi:10.1016/j.tem.2009.11.004

3. Biondi B. Thyroid and obesity: an intriguing relationship. J Clin Endocrinol Metab. 2010;95(8):3614-3617. doi:10.1210/jc.2010-1245

4. Karmisholt J, Andersen S, Laurberg P. Weight loss after therapy of hypothyroidism is mainly caused by excretion of excess body water associated with myxoedema. J Clin Endocrinol Metab. 2011;96(1): E99-E103. doi:10.1210/jc.2010-1521

5. O'Malley B, Hickey J, Nevens E. Thyroid dysfunction-weight problems and the psyche: the patientes'perspective. J Hum Nutr Diet. 2000;13:243-248. doi:10.1046/j.1365-277x.2000.00238.x

6. Plummer WA. Body weight in spontaneous myxedema; in American Association for the Study of Goiter: transactions of the American Association for the Study of Goiter. Rochester West J Surg Obstet Gynecol. 1940;88-98.

7. Garber JR, Cobin RH, Gharib H, et al. Clinical practice guidelines for hypothyroidism in adults: cosponsored by the American Association of Clinical Endocrinologists and the American Thyroid Association. Thyroid. 2012;22(12):1200-1235. doi:10.1089/thy.2012.0205 
8. Amouzegar A, Kazemian E, Abdi H, et al. Association between thyroid function and development of different obesity phenotypes in euthyroid adults: a nine-year follow-up. Thyroid. 2018;28(4):458464. doi:10.1089/thy.2017.0454

9. Ross DS, Burch HB, Cooper DS, et al. 2016 American Thyroid Association Guidelines for diagnosis and management of hyperthyroidism and other causes of thyrotoxicosis. Thyroid. 2016;26 (10):1343-1421. doi:10.1089/thy.2016.0229

10. Nordyke RA, Glibert FI, Harada ASM. Graves' disease. Influence of age on clinical findings. Arch Interm Med. 1988;148:626-631. doi:10.1001/archinte.1988.00380030132023

11. Vanderpump MP, Tunbridge WM. The epidemiology of thyroid diseases. In: Braverman LE, Utiger RD, editors. The Thyroid: $A$ Fundamental and Clinical Text. 8th ed. Philadelphia: Lippincott Williams and Wilkins; 2000:467.

12. Jonklaas J, Nsouli-Maktabi H. Weight changes in euthyroid patients undergoing thyroidectomy. Thyroid. 2011;21(12):1343-1351. doi:10.1089/thy.2011.0054

13. Rathi MS, Miles JN, Jennings PE. Weight gain during the treatment of thyrotoxicosis using conventional thyrostatic treatment. $J$ Endocrinol Invest. 2008;31(6):505-508. doi:10.1007/BF03346398

14. Hoermann R, Midgley J. TSH measurement and its implications for personalised clinical decision-making. J Thyroid Res. 2012;2012:1-9. Art. ID 438037. doi:10.1155/2012/438037
15. Crocker MK, Kaplowitz P. Treatment of paediatric hyperthyroidism but not hypothyroidism has a significant effect on weight. Clin Endocrinol (Oxf). 2010;73:752-759. doi:10.1111/j.1365-2265.2010.03877.x

16. Verma A, Jayaraman M, Kumar HKVS, Modi KD. Hypothyroidism and obesity: cause or effect? Saudi Med J. 2008;29(8):1135-1138.

17. Mamtani M, Kulkarni H, Dyer TD, et al. Increased waist circumference is independently associated with hypothyroidism in Mexican Americans: replicative evidence from two large, population-based studies. BMC Endocr Disord. 2014;14(46):1-13. doi:10.1186/14726823-14-1

18. De Moura Souza A, Sichieri R. Association between serum TSH concentration within the normal range and adiposity. Eur $J$ Endocrinol. 2011;165:11-15. doi:10.1530/EJE-11-0261

19. Reihner T. Obesity and thyroid function. Mol Cell Endocrinol. 2010;316:165-171. doi:10.1016/j.mce.2009.06.005

20. Greenlund LJ, Nair KS, Brennan MD. Changes in body composition in women following treatment of overt and subclinical hyperthyroidism. Endocr Pract. 2008;14(8):973-978. doi:10.4158/EP.14.8.973

21. Dutta P, Bhansali A, Walia R, Khandelwal N, Das S, Masoodi SR. Weight homeostasis and its modulators in hyperthyroidism before and after treatment with carbimazole. Indian $J$ Med Res. 2012;136:242-248.
International Journal of General Medicine

\section{Publish your work in this journal}

The International Journal of General Medicine is an international, peer-reviewed open-access journal that focuses on general and internal medicine, pathogenesis, epidemiology, diagnosis, monitoring and treatment protocols. The journal is characterized by the rapid reporting of reviews, original research and clinical studies

\section{Dovepress}

across all disease areas. The manuscript management system is completely online and includes a very quick and fair peer-review system, which is all easy to use. Visit http://www.dovepress.com/ testimonials.php to read real quotes from published authors. 